\title{
A theory-based online health behaviour intervention for new university students (U@Uni:LifeGuide): results from a repeat randomized controlled trial
}

David Cameron ${ }^{1}$, Tracy Epton ${ }^{2}$, Paul Norman ${ }^{1 *}$, Paschal Sheeran ${ }^{3}$, Peter R. Harris ${ }^{4}$, Thomas L. Webb', Steven A. Julious ${ }^{5}$, Alan Brennan ${ }^{5}$, Chloe Thomas ${ }^{5}$, Andrea Petroczi ${ }^{6}$, Declan Naughton ${ }^{6}$ and Iltaf Shah ${ }^{6}$

\begin{abstract}
Background: This paper reports the results of a repeat trial assessing the effectiveness of an online theory-based intervention to promote healthy lifestyle behaviours in new university students. The original trial found that the intervention reduced the number of smokers at 6-month follow-up compared with the control condition, but had non-significant effects on the other targeted health behaviours. However, the original trial suffered from low levels of engagement, which the repeat trial sought to rectify.

Methods: Three weeks before staring university, all incoming undergraduate students at a large university in the UK were sent an email inviting them to participate in the study. After completing a baseline questionnaire, participants were randomly allocated to intervention or control conditions. The intervention consisted of a self-affirmation manipulation, health messages based on the theory of planned behaviour and implementation intention tasks. Participants were followed-up 1 and 6 months after starting university. The primary outcome measures were portions of fruit and vegetables consumed, physical activity levels, units of alcohol consumed and smoking status at 6-month follow-up.
\end{abstract}

Results: The study recruited 2,621 students (intervention $n=1346$, control $n=1275$ ), of whom 1495 completed at least one follow-up (intervention $n=696$, control $n=799$ ). Intention-to-treat analyses indicated that the intervention had a non-significant effect on the primary outcomes, although the effect of the intervention on fruit and vegetable intake was significant in the per-protocol analyses. Secondary analyses revealed that the intervention had significant effects on having smoked at university (self-report) and on a biochemical marker of alcohol use.

Conclusions: Despite successfully increasing levels of engagement, the intervention did not have a significant effect on the primary outcome measures. The relatively weak effects of the intervention, found in both the original and repeat trials, may be due to the focus on multiple versus single health behaviours. Future interventions targeting the health behaviour of new university students should therefore focus on single health behaviours.

Trial registration: Current Controlled Trials ISRCTN07407344.

Keywords: Binge drinking, Diet, Exercise, Implementation intentions, Internet, Self-affirmation, Smoking, Students, Theory of planned behaviour, Young people

\footnotetext{
*Correspondence: p.norman@sheffield.ac.uk

'Department of Psychology, University of Sheffield, Western Bank, Sheffield S10 2TP, UK

Full list of author information is available at the end of the article
}

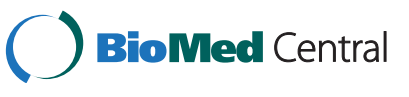

(c) 2015 Cameron et al. Open Access This article is distributed under the terms of the Creative Commons Attribution 4.0 International License (http://creativecommons.org/licenses/by/4.0/), which permits unrestricted use, distribution, and reproduction in any medium, provided you give appropriate credit to the original author(s) and the source, provide a link to the Creative Commons license, and indicate if changes were made. The Creative Commons Public Domain Dedication waiver (http://creativecommons.org/publicdomain/zero/1.0/) applies to the data made available in this article, unless otherwise stated. 


\section{Background}

The performance of health-promoting behaviours (e.g., eating fruit and vegetables, engaging in regular exercise), coupled with the avoidance of health-risk behaviours (e.g., excessive alcohol consumption, smoking), is important in reducing the risk of developing serious health problems, such as cancer, cardiovascular disease, obesity and type 2 diabetes [1]. A recent survey in England indicated that among 16-24 year olds, only $17 \%$ of men and $19 \%$ of women ate at least five portions of fruit and vegetables per day, $83 \%$ of men but only $57 \%$ of women met recommended levels of weekly physical activity, $27 \%$ of men and $19 \%$ of women consumed more than double the recommended daily limit of alcohol in the previous week and $27 \%$ of men and $19 \%$ of women were current smokers $[2,3]$. Given that there is evidence of clustering of health behaviours $[4,5]$, it is likely that many young people engage in a number of healthcompromising behaviours, thereby placing themselves at increased risk of developing serious health problems. There is therefore a clear need for interventions that target multiple health behaviours in young people. Encouragingly, there is some evidence that such interventions can have positive effects on health behaviour [6-8].

An earlier trial [9] tested the efficacy of a theory-based online intervention (U@Uni) targeting four health behaviours (fruit and vegetable intake, physical activity, alcohol consumption, and smoking) during the transition from school to university. Such life transitions are ideal opportunities to intervene as they represent 'teachable' moments; times when people's social environments and supporting cues for behaviour are in a state of flux and people are therefore more amenable to change $[10,11]$. Moreover, given that more than 500,000 students and over $30 \%$ of 18 year olds in England enter higher education each year [12], interventions delivered during this transition have the potential to reach a large number of young people. The intervention used three theory-based techniques to target the four health behaviours. First, a self-affirmation manipulation was used to reduce defensive processing of health messages [13]. Second, theorybased messages were developed through formative research to target the key beliefs underlying the four health behaviours, in order to increase young people's motivation to engage in healthy behaviours [14]. Third, implementation intention tasks were included to help translate good intentions into healthy behaviour [15].

The intervention had a significant effect on smoking, with fewer current smokers at follow-up in the intervention than in the control condition, although the intervention did not significantly affect the other three primary outcomes (i.e., fruit and vegetable intake, physical activity, alcohol consumption) [16]. Despite these largely non-significant effects, the health economic modelling revealed that rolling out the intervention to other universities would be likely to be cost-effective, primarily because of the low cost of the intervention and the impact of reduced smoking on future health outcomes [17].

Unfortunately, the trial was compromised by a number of limitations, which resulted in low levels of engagement with the intervention. Only $52 \%$ of participants allocated to the intervention condition completed the self-affirmation task, only $35 \%$ accessed the health messages and only $1 \%$ formed an implementation intention. As a result, it is difficult to determine whether the nonsignificant results are due to failure of theory or failure of intervention fidelity caused by low engagement. In addition, low engagement is also likely to lead to an inaccurate estimate of the effect of the intervention on the health behaviours, which an expected value of information analysis identified as an important driver of decision uncertainty in the health economic modelling [17].

There were three potential reasons for low engagement. First, the baseline questionnaire was timeconsuming to complete (approximately 20 minutes) due to the large number of items needed to assess the primary and secondary outcome variables. Having completed the questionnaire, many participants may have been fatigued and less inclined to proceed to, and engage with, the intervention. Second, the bespoke software platform that was developed to deliver the intervention had a number of technical glitches, meaning that participants' experience of completing the baseline questionnaire and engaging with the intervention was suboptimal. In particular, what was intended as a seamless process was experienced as a series of discrete steps with subsequent drop-outs at each step. For example, after completing the baseline questionnaire, participants in the intervention condition were directed to the U@Uni log-in page where they had to enter some registration details before completing the selfaffirmation manipulation. After completing the selfaffirmation manipulation, participants had to $\log$ in again to access the intervention material, and many failed to do so. Third, participants in the intervention condition had complete control over the amount and type of intervention material that they viewed. For example, participants in the intervention condition could choose which health behaviours and which belief-based messages to view and whether or not to make plans. This is also likely to have reduced engagement, as participants could simply choose not to view any messages or make any plans.

With these limitations in mind, a repeat trial was conducted with a number of changes designed to increase engagement with the intervention and provide a more accurate estimate of the efficacy of the intervention. 
First, the baseline questionnaire was shortened. In particular, shorter and simpler measures of fruit and vegetable intake and alcohol consumption were included, and some secondary outcome measures (e.g., selfefficacy, perceived control) were removed. Second, the intervention was delivered using the LifeGuide opensource software platform [18]. LifeGuide has been specifically designed for researchers to develop, deliver and evaluate online health behaviour interventions. It allows participants to complete baseline measures, be randomly allocated to conditions and access intervention material, all within the same website. As a result, participants experience the various tasks as seamless, with reduced opportunity to exit between tasks. Third, the key content of the intervention was delivered in a more structured format so that participants could quickly access health messages and make plans for all four health behaviours. In particular, participants in the intervention condition worked through four short modules that required them to read at least one belief-based message and make at least one plan for each health behaviour, before gaining access to the full intervention website.

This paper reports the results of the repeat randomized controlled trial of a theory-based online health behaviour intervention delivered during the transition from school to university. The primary research question was whether the intervention produces significant changes in the health behaviours of new students (i.e., fruit and vegetable intake, physical activity, alcohol consumption and smoking status) at a 6-month followup. Additional research questions focused on whether the intervention (i) changes theory of planned behaviour variables (and whether these changes mediate the effect of the intervention on the health behaviours), (ii) enhances health status, (iii) reduces health service usage, (iv) reduces recreational drug use and (v) reduces body mass index (BMI).

\section{Methods}

\section{Participants and procedure}

Three weeks before starting university (in September 2013), all incoming undergraduate students to the University of Sheffield $(N=5,453)$ were sent an email inviting them to take part in the study, with a link to an online questionnaire containing measures of demographics, health status, intentions, and health behaviour. There were no exclusion criteria. Participants indicated their consent to participate by clicking a button on the first page before they were permitted to proceed to the rest of the questionnaire. Participants $(N=2,621$; mean age $=18.80$ years; $55 \%$ women) then completed the baseline questionnaire and were randomly allocated to the intervention $(n=1,346)$ and control $(n=1,275)$ conditions using the random function on LifeGuide [18].
Figure 1 shows the flow of participants through the trial and Table 1 provides details of the sample at baseline in terms of demographics and the primary and secondary outcomes. In addition, the proportions of the sample meeting guidelines for the four health behaviours at baseline are reported in Table 2, along with data for 1624-year-olds from the Health Survey for England [2, 3].

After completing the baseline questionnaire, participants assigned to the intervention condition were asked to complete a 'profile' page that contained the selfaffirmation manipulation. They were then directed to complete four short modules on each of the four health behaviours targeted by the intervention that contained theory-based messages and planning exercises. After completing all four modules, intervention participants in the intervention condition had access to the full website with further health messages and links on each of the four targeted health behaviours.

All participants were sent emails inviting them to complete follow-up questionnaires 1 month (October 2013) and 6 months (March 2014) after starting university. Participants were entered into a $£ 100$ prize draw as an incentive for completing each questionnaire. In addition, participants completing all three questionnaires received a $£ 10$ gift voucher and were entered into a further prize draw for an iPad Mini.

Participants were also sent emails when they started university, inviting them to provide additional data on the biochemical markers of health behaviour. A sample of 213 students (intervention, $n=90$; control, $n=123$; mean age $=18.93$ years; standard deviation $(\mathrm{SD})=2.76$ ) was recruited at baseline, of whom 133 also provided a hair sample when invited at 6-month follow-up (intervention, $n=63$; control, $n=70$ ).

Ethical approval for the study was obtained from the Department of Psychology Research Ethics Committee at the University of Sheffield.

\section{Intervention materials}

The self-affirmation manipulation was adapted from an existing value-affirmation task [19] and embedded in a 'profile' page. Participants were asked to provide details including their name, course, home town and main interests or hobbies, before being presented with a list of eight commonly held personal values (sense of humour, academic achievement, relations with family and friends, social skills, spontaneity, artistic skills or aesthetic appreciation, religion, faith or spirituality, and respect, decency or manners). Participants were asked to select their most important value (or provide their own) and to explain briefly why the value was important to them. The resultant information formed part of the user's 'profile, which was displayed in the banner at the top of all pages of the intervention website that included the 


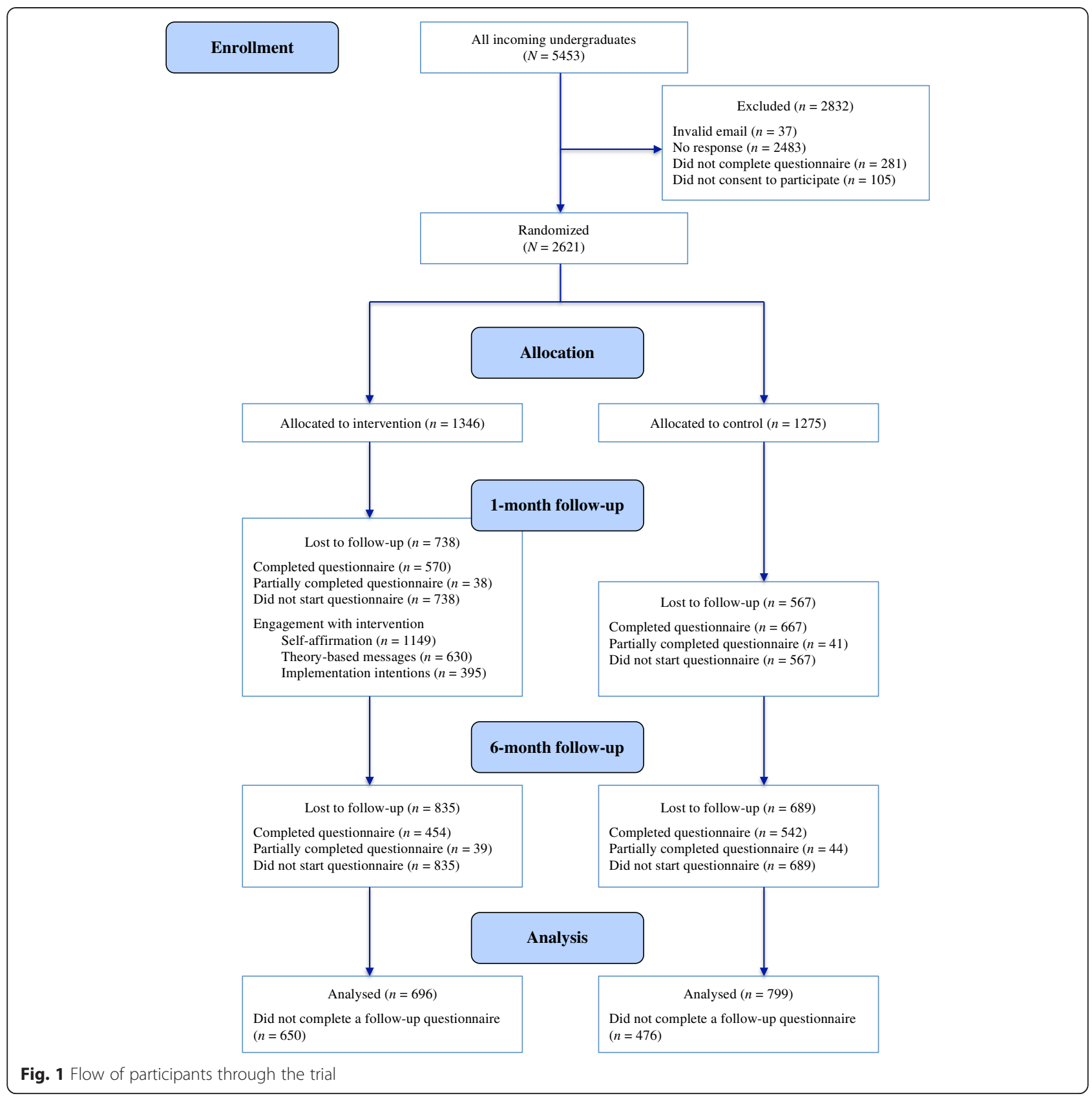

participant's name, the value that they chose and the reason why it was important to them ('I value $\mathrm{X}$ because Y.').

After completing the self-affirmation manipulation, participants were directed to complete short modules on each of the four targeted health behaviours. Theory-based messages were developed to encourage adequate fruit and vegetable intake and regular exercise, and to discourage binge drinking and smoking. The messages were based on the theory of planned behaviour [20] and developed on the basis of formative work that identified the key behavioural, normative and control beliefs associated with intentions to perform each of the four health behaviours in new university students [14].

Module 1 focused on exercising regularly at university. Participants were presented with a list of topics that targeted key beliefs from the formative research and instructed to choose one (e.g., 'Exercise improves your fitness'). They were then directed to a webpage containing theory-based messages (i.e., text), videos of students talking about the targeted belief, and links to other related material. After viewing the page, participants had the opportunity to either view another topic or message or proceed to the planner. 
Table 1 Characteristics of the sample at baseline

\begin{tabular}{|c|c|c|c|c|c|c|c|}
\hline \multirow[t]{2}{*}{ Variable } & & \multicolumn{3}{|l|}{ Control } & \multicolumn{3}{|l|}{ Intervention } \\
\hline & & $\%$ or mean & Standard deviation & $n$ & $\%$ or mean & Standard deviation & $n$ \\
\hline \multicolumn{8}{|c|}{ Demographics } \\
\hline \multirow[t]{2}{*}{ Nationality } & UK & 75.37 & - & 961 & 78.16 & - & 1052 \\
\hline & Non-UK & 24.63 & - & 314 & 21.84 & - & 294 \\
\hline \multirow[t]{7}{*}{ Ethnicity } & White British & 68.67 & - & 835 & 71.65 & - & 930 \\
\hline & White other & 7.40 & - & 90 & 6.32 & - & 82 \\
\hline & Mixed & 3.87 & - & 47 & 3.70 & - & 48 \\
\hline & Asian and Asian British & 5.10 & - & 62 & 5.32 & - & 69 \\
\hline & Black and Black British & 206 & - & 25 & 2.77 & - & 36 \\
\hline & Chinese & 10.20 & - & 124 & 8.24 & - & 107 \\
\hline & Other & 2.71 & - & 33 & 2.00 & - & 26 \\
\hline \multirow[t]{2}{*}{ Sex } & Female & 54.87 & - & 698 & 55.81 & - & 749 \\
\hline & Male & 45.13 & - & 574 & 44.19 & - & 593 \\
\hline \multicolumn{2}{|l|}{ Age } & 18.89 & 2.68 & 1274 & 18.73 & 2.01 & 1340 \\
\hline \multicolumn{8}{|c|}{ Fruit and vegetable intake } \\
\hline \multicolumn{2}{|c|}{ Portions per day } & 4.48 & 2.21 & 1267 & 4.49 & 2.34 & 1344 \\
\hline \multicolumn{8}{|c|}{ Physical activity } \\
\hline \multicolumn{2}{|c|}{ Metabolic equivalent of task per week } & 3665.30 & 3518.61 & 1273 & 3510.02 & 3276.63 & 1343 \\
\hline \multicolumn{8}{|c|}{ Alcohol consumption } \\
\hline \multicolumn{2}{|c|}{ Total units the in previous 7 days } & 6.77 & 9.32 & 1271 & 7.15 & 9.72 & 1344 \\
\hline \multicolumn{2}{|c|}{ Number of days binge drinking in the last 7 days (drinkers only) } & 0.52 & 0.80 & 833 & 0.56 & 0.82 & 872 \\
\hline \multicolumn{2}{|c|}{ Biochemical marker of alcohol consumption (fatty acid ethyl esters) } & 3.24 & 4.09 & 123 & 2.60 & 3.33 & 90 \\
\hline \multicolumn{8}{|l|}{ Smoking } \\
\hline \multicolumn{2}{|c|}{ Has smoked } & 42.89 & - & 1175 & 44.31 & - & 1248 \\
\hline \multicolumn{2}{|c|}{ Current smoker } & 3.49 & - & 1175 & 2.80 & - & 1248 \\
\hline \multicolumn{2}{|c|}{ Cigarettes smoked per week (smokers only) } & 49.05 & 38.93 & 41 & 53.60 & 45.95 & 35 \\
\hline \multicolumn{2}{|c|}{ Smoking marker (cotinine) } & 0.48 & 0.44 & 123 & 0.44 & 0.41 & 90 \\
\hline \multicolumn{2}{|c|}{ Smoking marker (nicotine) } & 3.89 & 10.32 & 123 & 6.52 & 17.83 & 90 \\
\hline \multicolumn{8}{|c|}{ Other outcomes } \\
\hline EQ-5D-3 & & & & & & & \\
\hline Health in & Q-5D-3 L (visual analogue scale) & 0.94 & 0.12 & 1249 & 0.94 & 0.12 & 1327 \\
\hline Health in & Q-5D-3 L (time trade off) & 0.95 & 0.11 & 1249 & 0.95 & 0.11 & 1327 \\
\hline EQ-5D-3 & scale & 83.26 & 12.12 & 1256 & 82.01 & 13.77 & 1326 \\
\hline Recreatic & & & & & & & \\
\hline Have tak & rugs (single sample count method) & 19.11 & 7.21 & 1275 & 17.84 & 6.79 & 1336 \\
\hline Have tak & rugs (biochemical marker) & 24.39 & - & 123 & 24.44 & - & 123 \\
\hline Body mass & & & & & & & \\
\hline Self-repo & & 21.78 & 3.69 & 1206 & 21.95 & 3.88 & 1274 \\
\hline Objective & & 21.67 & 3.55 & 123 & 21.5 & 3.39 & 90 \\
\hline Social cogn & & & & & & & \\
\hline Fruit and & tion & 4.78 & 1.59 & 1150 & 4.76 & 1.58 & 1258 \\
\hline Physical & & 5.72 & 1.38 & 1156 & 5.69 & 1.43 & 1263 \\
\hline Binge $d r$ & & 3.33 & 1.90 & 1156 & 3.32 & 1.92 & 1249 \\
\hline Smoking & & 1.51 & 1.29 & 1173 & 1.46 & 1.20 & 1269 \\
\hline
\end{tabular}


Table 2 Percentages of men and women meeting health behaviour guidelines in the baseline sample and among 16-24 years in England $[2,3]$

\begin{tabular}{|c|c|c|c|c|}
\hline \multirow[b]{2}{*}{ Variable } & \multicolumn{2}{|c|}{ Baseline sample } & \multicolumn{2}{|c|}{ Health Survey for England data (16-24-year-olds) } \\
\hline & Men & Women & Men & Women \\
\hline \multicolumn{5}{|l|}{ Fruit and vegetable intake } \\
\hline Five or more portions per day & 41 & 44 & 17 & 19 \\
\hline \multicolumn{5}{|l|}{ Physical activity } \\
\hline 150 minutes per week & 83 & 61 & 83 & 57 \\
\hline \multicolumn{5}{|l|}{ Alcohol consumption } \\
\hline Not more than 21 (men) or 14 (women) units per week & 88 & 91 & 80 & 83 \\
\hline \multicolumn{5}{|l|}{ Smoking } \\
\hline Current non-smoker & 3 & 3 & 27 & 19 \\
\hline
\end{tabular}

The planner helped participants to form implementation intentions by asking them to identify (i) a good opportunity to act on their intentions (e.g., when they have spare time between lectures) and (ii) a suitable response to their identified opportunity (e.g., to go swimming in the university pool) for each of the four targeted health behaviours. Participants were presented with an example of an implementation intention in an if-then format [21] (e.g., 'IF I am tempted to skip exercising, THEN I will tell myself 'no excuses' and remind myself that I will feel great after exercising'). Spaces were provided for participants to make up to three if-then plans by linking an identified opportunity and appropriate response. Participants were presented with their plan and asked to repeat it to themselves several times. A record of the plan was also automatically emailed to the participant.

When participants had finished Module 1, they were presented with the first page of Module 2 ('Eating fruit and vegetables'), and instructed to work through the module in the same way as for Module 1. Participants then completed Modules 3 ('Avoiding binge drinking') and 4 ('Avoiding smoking'). When all four modules had been completed, participants had access to the full website, containing messages targeting all of the key beliefs from the formative research, links to the planner, saved plans and general health information. All participants completed the modules in the same order as described.

\section{Measures}

The four primary outcome measures were (i) the number of portions of fruit and vegetables consumed per day, (ii) physical activity in the previous week, (iii) alcohol consumption in the previous week, and (iv) smoking status at 6-month follow-up. A range of secondary outcome measures was also assessed as detailed next. Unless indicated, all of the measures were taken at baseline as well as at 1- and 6-month follow-up.

\section{Fruit and vegetable intake}

Fruit and vegetable intake (portions per day) was measured using a two-item dietary questionnaire [22], which has been validated against biochemical markers (e.g., potassium excretion, urinary potassium:creatinine ratio and plasma concentration of vitamin C). Participants were asked to report the amount of (i) fruit and (ii) vegetables consumed in a typical day (at baseline) and since starting university (at 1 and 6-month follow-up). Reponses to the items were summed to give an estimated total of daily fruit and vegetable consumption.

\section{Physical activity}

The Short Form of International Physical Activity Questionnaire was used to assess levels of physical activity [23]. This questionnaire has undergone extensive testing across 12 countries and evidence attests to its reliability and validity. Participants were asked to indicate how many times, and for how long, they had engaged in vigorous exercise (defined as 'activities that take hard physical effort and make you breathe much harder than normal'), moderate exercise (defined as 'activities that take moderate physical effort and make you breathe somewhat harder than normal') and walking in the previous 7 days. Responses were converted into 'metabolic equivalents of task', to provide a total score representing the total amount of physical activity over the 7 days.

\section{Alcohol}

Alcohol consumption was assessed using a retrospective 7-day recall drinking diary, in which participants reported the amount of alcohol (units) consumed on each of the previous 7 days [24]. The total number of units of alcohol consumed in the previous week and the number of binge sessions were calculated. The Alcohol Use Disorders Identification Test (AUDIT) [25] was also included at 6-month follow-up, to assess hazardous and harmful patterns of alcohol use. 


\section{Smoking}

Items based on the Health Survey for England [2] were used to assess participants' current smoking status and the typical number of cigarettes or amount of tobacco that they smoked. In addition, at 1 and 6-month followup, participants were asked, 'Since starting university have you smoked at all (even a puff or just socially)?'

\section{Health status}

The EQ-5D-3 L [26], a short standardized measure of health status, was used to assess levels of severity (no problems, some or moderate problems, extreme problems) in five domains: mobility, self-care, usual activities, pain or discomfort, and anxiety or depression. The EQ5D-3 L provides a descriptive profile and a single index value for health status and is recommended as the measure of health-related quality of life for health economic evaluations in the UK [27]. The EQ-5D-3 L was assessed at baseline and 6-month follow-up.

\section{Recreational drug use}

A single sample count method [28] was used to estimate the prevalence of recreational drug use in the sample. Respondents were asked to indicate the number of 'yes' answers $(0$ or $5,1,2,3,4)$ to five questions - four of which have a $50 \%$ population prevalence (e.g., odd or even date of birth) and one of which was about their use of recreational drugs. The position of the sensitive item (i.e., 'I have used recreational drugs in the last 3 months/ since starting university') was randomized. The single sample count method can be used to provide an estimate of the prevalence of recreational drug use in the sample without being able to identify whether individual participants do or do not use recreational drugs, on the basis that $50 \%$ of the sample should answer 'yes' answers to each of the four non-sensitive questions. This method has been shown to encourage accurate reporting of behaviours that are illegal and could be regarded as socially undesirable [29].

\section{Body mass index}

All participants recorded their height and weight, from which their BMI was calculated. Those participants who provided a hair sample for analysis also had their height and weight measured to provide an objective measure of BMI.

\section{Health services usage}

Participants were asked to report their use of health services (e.g., general practitioner visits, hospitalizations) at the 6-month follow-up.

\section{Social cognitive variables}

Single-item measures of social cognitive variables for each behaviour were included. Intentions (e.g., 'Do you intend to engage in regular exercise at university?') were measured at all three time points. Affective attitudes (e.g., 'Engaging in regular exercise at university would be... unpleasant/pleasant'), cognitive attitudes (e.g., 'Engaging in regular exercise at university would be... harmful/beneficial'), subjective norms (e.g., 'Most people who are important to me think I should/should not engage in regular exercise at university'), descriptive norms (e.g., 'Most students will engage in regular exercise at university'), self-efficacy (e.g., 'If I wanted, I could easily engage in regular exercise at university'), perceived control (e.g., 'How much control do you have over whether or not you engage in regular exercise at university?'), and planning (e.g., 'To what extent do you have a clear plan of how to engage in regular exercise at university?') were assessed at the 1- and 6-month follow-ups.

\section{Engagement with the intervention}

Engagement with the intervention was measured by identifying whether or not participants (i) completed the self-affirmation task (i.e., profile page), (ii) viewed the theory-based messages in the four modules and (iii) formed implementation intentions for the four health behaviours.

\section{Biochemical measures}

Hair samples $(3 \mathrm{~cm}$ long) were liquefied and analyzed for biochemical markers of alcohol consumption, cigarette smoking and recreational drug use. Following extraction procedures, markers of alcohol (fatty acid ethyl esters) and cigarettes (nicotine, cotinine) were quantified using liquid chromatography with tandem mass spectrometric detection. In addition, evidence for recreational drug use was detected by screening for commonly used drugs and their metabolites. These included: amphetamine, 3,4-methylenedioxyamphetamine, 3,4-methylenedioxy- $N$-methylamphetamine, ephedrine, mephedrone, tetrahydrocannabinol, cocaine, heroin, lysergic acid diethylamide, phencyclidine and ketamine. Morphine, codeine, hydromorphone and hydrocodone were treated separately owing to their potential medical use (i.e., as a pain reliever or cough suppressant). A 6430 triple quadruple mass spectrometer (Agilent Technologies UK) was employed, with a dynamicmultiple reaction monitoring-liquid chromatography mass spectrometry method.

\section{Statistical analysis}

The original trial of the $\mathrm{U} @ U$ Uni intervention [16] achieved an initial response rate of $31.34 \%$ to the recruitment emails, and obtained follow-up data for at 
least one time point from $76.60 \%$ of respondents. $A$ priori sample size calculations for the repeat trial indicated that, assuming the same response and retention rates, a total sample size of approximately $5000 \times$ $0.3134 \times 0.7660=1200$ (i.e., 600 per arm of the trial) would be obtained that would be sufficient to detect a standardized effect size of $d=0.20$ at a two-sided significance level of 0.0127 with $80 \%$ power. Webb et al. [8] reported that the overall effect size of internet-based health behaviour interventions was $d=0.16$, although this increased for interventions based on the theory of planned behaviour $(d=0.36)$ and using implementation intentions $(d=0.25)$. For the hair analysis, assuming the same response and retention rates as in the original trial, it was estimated that a final sample of 84 would be obtained that would be sufficient to detect a medium effect size of $d=0.62$ (alpha $=0.05$, power $=0.80)$.

The data analysis plan for the repeat trial was the same as for the original trial [16] and as reported in the study protocol [9]. Analyses assessing intervention effects on the primary outcome variables (i.e., the four targeted health behaviours at 6-month follow-up) were conducted using an intention-to-treat approach (i.e., data were included from all participants who completed at least one follow-up survey). Missing data at 6-months were imputed from the 1-month follow-up data by carrying the last observation forward [30, 31]. A series of analyses of covariance (ANCOVAs) and logistic regression analyses were used to assess the impact of the intervention on performance of the targeted behaviours at 6-month follow-up, controlling for corresponding baseline scores, sex, age and nationality (i.e., UK or non-UK). For primary outcomes, the Bonferroni correction was used to account for multiple tests. Thus, statistical significance was declared if any of the primary endpoints were significant at 0.0127 . The primary analyses were repeated without imputing data, as recommended by Altman [32]. Further analyses were conducted to assess the effect of variables that might moderate the effect of the intervention on the primary outcomes, including sex, nationality, ethnicity and engagement with the intervention (per-protocol analyses).

The impact of the intervention on secondary outcomes (i.e., health behaviours at 1 month follow-up, social cognitive variables, health status, recreational drug use, BMI, health services usage, and biochemical measures) was assessed using a similar analysis strategy (i.e., using ANCOVAs and logistic regression analyses that controlled for corresponding baseline scores (where available), sex, age and nationality). No adjustments were made for multiple tests, and intention-to-treat analyses were not performed for the secondary outcomes. Possible adverse consequences of the intervention (i.e., harms) were assessed by considering effects on the primary outcomes and key secondary outcomes (i.e., recreational drug use, AUDIT scores, health status, health services usage).

In addition, analyses were conducted to compare students who did or did not participate in the trial in response to the invitation email on demographics (age, sex, nationality), as well as participants who were randomly allocated to the intervention or control conditions (randomization check), participants who did or did not provide a hair sample, and participants who did or did not complete a follow-up questionnaire (attrition analyses), on the baseline measures using independent sample $t$ tests (for continuous variables) and chi-square tests (for categorical variables).

\section{Results}

\section{Randomization checks}

There were no significant differences between participants in the intervention and control conditions on any of the baseline measures (see Table 1).

\section{Comparison between participants who provided or who did not provide a hair sample}

No significant differences were found between participants who provided or did not provide a hair sample on the primary outcome variables at baseline.

\section{Attrition analyses}

Comparing the demographic profile of students who did or did not participate in the trial in response to the invitation email revealed that women were more likely to participate in the trial than men $(54.1 \%$ vs. $42.0 \%)$, $\chi^{2}(1, N=5451)=79.32, P<0.001$, as were non-UK students versus UK students (57.8 \% vs. $45.7 \%), \chi^{2}(1, N=$ $5453)=49.65, P<0.001$. Students who participated in the trial were also slightly younger than those who did not $($ mean $=18.76, \quad \mathrm{SD}=2.44$ vs. mean $=18.95, \mathrm{SD}=$ 2.42), $t(5451)=2.96, P=0.003$.

Examining attrition after baseline revealed that participants who completed at least one follow-up questionnaire differed from those who did not complete a follow-up questionnaire in nationality, $X^{2}$ $(1, N=2621)=23.18, P<0.001$, ethnicity, $\chi^{2} \quad(1, N=$ $2514)=11.39, P<0.001$, sex, $X^{2}(1, N=2621)=33.47$, $P<0.001$, BMI, $t(2478)=2.48, P=0.013$, and baseline intentions to consume fruit and vegetables, $t(2406)=$ 2.38, $P=0.017$. Completers were more likely to be British, white and female, with a higher BMI and weaker intention to consume fruit and vegetables, than those who did not complete a follow-up questionnaire. In addition, there was a significant difference in drop-out rates between the two conditions, $x^{2}$ $(1, N=2621)=33.47, P<0.001 \quad(47.5 \%$ intervention, $36.3 \%$ control). 


\section{Primary outcomes}

There were no statistically significant differences between the intervention and control conditions on the primary outcomes at 6-month follow-up, although the effect of the intervention on fruit and vegetable intake approached significance: fruit and vegetable intake $(P=0.024)$, physical activity $(P=0.932)$, smoking status $(p=0.293)$, and alcohol consumption $(P=$ $0.277)$. Repeating the primary analyses without data imputation produced consistent results.

The effect sizes found in the repeat trial were comparable to those found in the original trial (see Table 3 ) for fruit and vegetable intake, $Q(1)=2.93, P=0.087$, physical activity, $Q(1)=0.00015, P=0.990$, alcohol consumption, $Q(1)=0.25, P=0.619$, and smoking status, $Q(1)=3.46$, $P=0.063$. However, two of the differences approached significance. A marginally larger effect size was found for fruit and vegetable intake in the repeat trial $(d=$ $0.12)$ than in the original trial $(d=-0.02)$, whereas a marginally larger effect size was found for smoking status in the original trial $(d=0.25)$ than in the repeat trial $(d=0.10)$.

\section{Moderation analysis}

Sex, nationality (UK vs. non-UK), and ethnicity (white vs. non-white) did not moderate the effect of the intervention on any of the primary outcome variables.

\section{Engagement}

Of the 1,346 participants allocated to the intervention condition, 1,149 (85\%) completed the self-affirmation task. Considering engagement with the health messages, 973 participants $(72 \%)$ viewed a message for at least one behaviour, $672(50 \%)$ for at least two behaviours, 640 (48\%) for at least three behaviours, and 630 (47\%) for all four behaviours. Considering engagement with the planning tasks, 554 participants $(41 \%)$ formed an implementation intention for at least one behaviour, 479 (36 \%) for at least two behaviours, 439 (33\%) for at least three behaviours, and 395 (29\%) for all four behaviours.

\section{Per-protocol analysis}

To assess the effect of engagement with the intervention on the primary outcomes, per-protocol analyses were conducted that included all participants in the control condition $(N=799)$ and, for each health behaviour, only those participants in the intervention condition who completed the self-affirmation profile, viewed a health message and formed an implementation intention $(N=$ 281 for fruit and vegetable intake, 297 for physical activity, 253 for alcohol consumption, and 238 for smoking). These analyses revealed that participants in the intervention condition who had engaged with the intervention reported consuming significantly more portions of fruit and vegetables, $F(1,1068)=7.19, P=0.007$, than those in the control condition ( mean $=4.23,3.89$; $\mathrm{SD}=0.11$ and 0.07 , respectively). Like the primary analyses, the per-protocol analyses revealed no significant effect of the intervention on levels of physical activity, $F$ (1, $1079)=0.80, P=0.371$, units of alcohol consumed, $F(1,1030)=1.30, P=0.254$, or current smoking status, $B=-0.33, \mathrm{SD}=0.33, P=0.332$.

\section{Secondary outcomes}

The intervention was found to have a number of significant effects on secondary outcomes (see Table 4). In particular, the intervention had a significant effect on smoking at university at 6-month follow-up, such that $37.16 \%$ of participants in the control condition reported that they had smoked since starting university compared with only $30.70 \%$ of participants in the intervention condition. In addition, the intervention had a significant

Table 3 Estimated marginal means, percentages, sample sizes, standard deviations and $P$ values for primary outcomes at 6-month follow-up in the original and repeat trials

\begin{tabular}{|c|c|c|c|c|c|c|c|c|c|c|c|c|c|c|c|c|}
\hline \multirow[t]{3}{*}{ Variable } & \multicolumn{8}{|c|}{ Original trial } & \multicolumn{8}{|c|}{ Repeat trial } \\
\hline & \multicolumn{3}{|l|}{ Control } & \multicolumn{5}{|c|}{ Intervention } & \multicolumn{3}{|l|}{ Control } & \multicolumn{5}{|c|}{ Intervention } \\
\hline & $\begin{array}{l}\% \text { or } \\
\text { mean }\end{array}$ & SD & $n$ & $\begin{array}{l}\% \text { or } \\
\text { mean }\end{array}$ & SD & $n$ & $p$ & $d$ & $\begin{array}{l}\% \text { or } \\
\text { mean }\end{array}$ & SD & $n$ & $\begin{array}{l}\% \text { or } \\
\text { mean }\end{array}$ & SD & $n$ & $p$ & $d$ \\
\hline \multicolumn{17}{|c|}{ Fruit and vegetable intake } \\
\hline Portions per day & 5.72 & 4.98 & 512 & 5.61 & 4.89 & 495 & 0.708 & -0.02 & 3.89 & 1.97 & 793 & 4.11 & 1.84 & 690 & 0.024 & 0.12 \\
\hline \multicolumn{17}{|l|}{ Physical activity } \\
\hline $\begin{array}{l}\text { Metabolic } \\
\text { equivalent of task }\end{array}$ & 3316.10 & 5143.79 & 526 & 3350.52 & 5144.16 & 513 & 0.914 & 0.01 & 3613.27 & 2578.07 & 788 & 3627.94 & 2578.97 & 671 & 0.932 & 0.01 \\
\hline \multicolumn{17}{|l|}{ Alcohol consumption } \\
\hline Units in last 7 days & 13.41 & 19.65 & 547 & 13.10 & 19.75 & 540 & 0.737 & 0.02 & 11.03 & 10.91 & 782 & 10.42 & 10.86 & 668 & 0.277 & 0.06 \\
\hline \multicolumn{17}{|l|}{ Smoking } \\
\hline Current smoker & 13.02 & - & 553 & 8.70 & - & 540 & 0.010 & 0.25 & 14.05 & - & 783 & 11.18 & - & 671 & 0.293 & 0.10 \\
\hline
\end{tabular}


Table 4 Estimated marginal means, percentages, sample sizes, standard deviations and $\mathrm{p}$ values for secondary outcomes

\begin{tabular}{|c|c|c|c|c|c|c|c|c|c|c|c|c|c|c|}
\hline \multirow{3}{*}{ Variable } & \multicolumn{8}{|c|}{ 1-month follow-up } & \multicolumn{6}{|c|}{ 6-month follow-up } \\
\hline & Contro & & & Interve & tion & & & & Control & & Intervention & & & \\
\hline & $\begin{array}{l}\% \text { or } \\
\text { mean }\end{array}$ & SD & $n$ & $\begin{array}{l}\% \text { or } \\
\text { mean }\end{array}$ & SD & $n$ & $p$ & $\begin{array}{l}d \\
\text { (odds } \\
\text { ratio) }\end{array}$ & $\begin{array}{l}\% \text { or } S D \\
\text { mean }\end{array}$ & $n$ & $\begin{array}{l}\% \text { or } S D \\
\text { mean }\end{array}$ & $n$ & P & $\begin{array}{l}d \\
\text { (odds } \\
\text { ratio) }\end{array}$ \\
\hline
\end{tabular}

Fruit and vegetable intake

$\begin{array}{lllllllll}\text { Portions per day } & 3.63 & 1.84 & 691 & 3.84 & 1.95 & 595 & 0.041 & 0.11\end{array}$

Physical activity

Metabolic equivalent of task $\begin{array}{llllllll}3501.75 & 2348.35 & 690 & 3515.20 & 2348.97 & 579 & 0.919 & 0.01\end{array}$

Alcohol consumption

\begin{tabular}{|c|c|c|c|c|c|c|c|c|c|c|c|c|c|c|c|c|}
\hline Units in the last 7 days & 12.06 & 12.01 & 682 & 11.45 & 11.78 & 578 & 0.364 & 0.05 & & & & & & & & \\
\hline $\begin{array}{l}\text { Number of days binge } \\
\text { drinking in previous } 7 \text { days } \\
\text { (drinkers only) }\end{array}$ & 1.02 & 0.90 & 508 & 0.98 & 1.03 & 421 & 0.547 & 0.004 & 0.99 & 0.95 & 564 & 0.97 & 0.87 & 473 & 0.674 & 0.02 \\
\hline $\begin{array}{l}\text { Alcohol Use Disorders } \\
\text { Identification Test }\end{array}$ & & & & & & & & & 9.32 & 4.94 & 504 & 9.40 & 4.90 & 416 & 0.809 & -0.02 \\
\hline $\begin{array}{l}\text { Biochemical marker of } \\
\text { alcohol consumption (fatty } \\
\text { acid ethyl esters) }\end{array}$ & & & & & & & & & 7.29 & 7.85 & 70 & 5.00 & 4.33 & 63 & 0.038 & 0.35 \\
\hline \multicolumn{17}{|l|}{ Smoking } \\
\hline Has smoked & 51.90 & - & 684 & 52.50 & - & 581 & 0.876 & $(1.01)$ & 54.72 & - & 795 & 53.57 & - & 685 & 0.933 & (0.96) \\
\hline $\begin{array}{l}\text { Smoked since attending } \\
\text { university }\end{array}$ & 27.34 & - & 684 & 26.16 & - & 581 & 0.723 & $\begin{array}{l}-0.02 \\
(0.97)\end{array}$ & 37.16 & - & 783 & 30.70 & - & 671 & 0.016 & $\begin{array}{l}0.11 \\
(0.81)\end{array}$ \\
\hline Current smoker & 11.84 & - & 684 & 11.36 & - & 581 & 0.390 & $\begin{array}{l}-0.03 \\
(0.96)\end{array}$ & & & & & & & & \\
\hline $\begin{array}{l}\text { Cigarettes smoked per week } \\
\text { (smokers only) }\end{array}$ & 16.21 & 20.11 & 81 & 17.02 & 25.87 & 66 & 0.832 & -0.04 & 19.87 & 28.19 & 74 & 18.30 & 21.75 & 46 & 0.749 & 0.06 \\
\hline Smoking marker (cotinine) & & & & & & & & & 1.02 & 6.40 & 70 & 0.38 & 1.49 & 63 & 0.460 & 0.13 \\
\hline Smoking marker (nicotine) & & & & & & & & & 2.52 & 11.94 & 70 & 2.91 & 17.96 & 63 & 0.867 & 0.03 \\
\hline \multicolumn{17}{|l|}{ Other outcomes } \\
\hline \multicolumn{17}{|l|}{ EQ-5D-3 L } \\
\hline $\begin{array}{l}\text { Health index scores from } \\
\text { EQ-5D-3 L (visual analogue } \\
\text { scale) }\end{array}$ & 0.92 & 0.26 & 663 & 0.91 & 0.24 & 559 & 0.907 & -0.04 & 0.91 & 0.28 & 810 & 0.90 & 0.26 & 702 & 0.262 & -0.04 \\
\hline $\begin{array}{l}\text { Health index scores from } \\
\text { EQ-5D-3 L (time trade off) }\end{array}$ & 0.93 & 0.26 & 663 & 0.93 & 0.24 & 559 & 0.873 & 0.00 & 0.93 & 0.28 & 810 & 0.92 & 0.26 & 702 & 0.208 & -0.04 \\
\hline $\begin{array}{l}\text { EQ-5D-3 L visual analogue } \\
\text { scale }\end{array}$ & 78.61 & 13.97 & 669 & 79.47 & 14.07 & 569 & 0.285 & 0.06 & 79.16 & 12.98 & 763 & 78.98 & 13.07 & 657 & 0.791 & -0.01 \\
\hline \multicolumn{17}{|l|}{ Recreational drug use } \\
\hline $\begin{array}{l}\text { Have taken recreational } \\
\text { drugs (single sample count } \\
\text { method) }\end{array}$ & 4.84 & 8.83 & 708 & 4.00 & 9.61 & 608 & 0.450 & 0.11 & 23.18 & 11.18 & 586 & 21.78 & 12.00 & 493 & 0.556 & 0.04 \\
\hline $\begin{array}{l}\text { Have taken recreational } \\
\text { drugs (biochemical marker) }\end{array}$ & & & & & & & & & 55.71 & - & 70 & 52.38 & - & 63 & 0.717 & (0.88) \\
\hline \multicolumn{17}{|l|}{ Body mass index } \\
\hline Self-report & 21.63 & 1.70 & 591 & 21.52 & 1.77 & 491 & 0.302 & 0.06 & 22.00 & 2.38 & 698 & 21.84 & 2.44 & 596 & 0.231 & 0.07 \\
\hline Objective & & & & & & & & & 21.79 & 2.54 & 70 & 22.43 & 3.49 & 63 & 0.255 & -0.20 \\
\hline \multicolumn{17}{|l|}{ Health service usage } \\
\hline \multirow[t]{2}{*}{$\begin{array}{l}\text { Times visited general } \\
\text { practitioner in previous } \\
6 \text { months }\end{array}$} & & & & & & & & & 1.48 & 2.31 & 532 & 1.48 & 0.11 & 449 & 0.973 & 0.00 \\
\hline & & & & & & & & & 1.90 & - & 9 & 0.80 & - & 3 & 0.156 & 0.36 \\
\hline
\end{tabular}


Table 4 Estimated marginal means, percentages, sample sizes, standard deviations and p values for secondary outcomes (Continued)

\begin{tabular}{|c|c|c|c|c|c|c|c|c|c|c|c|c|c|c|c|c|}
\hline \multicolumn{17}{|l|}{$\begin{array}{l}\text { General practitioner offered } \\
\text { alcohol intervention }\end{array}$} \\
\hline $\begin{array}{l}\text { No alcohol intervention } \\
\text { offered }\end{array}$ & & & & & & & & & 98.1 & - & 463 & 99.2 & - & 394 & & $(1.92)$ \\
\hline $\begin{array}{l}\text { Attended alcohol } \\
\text { intervention }\end{array}$ & & & & & & & & & 66.7 & - & 2 & 0.00 & - & 0 & 0.889 & - \\
\hline $\begin{array}{l}\text { Did not attend alcohol } \\
\text { intervention }\end{array}$ & & & & & & & & & 33.3 & - & 4 & 100 & - & 2 & & - \\
\hline $\begin{array}{l}\text { Times visited accident and } \\
\text { emergency department }\end{array}$ & & & & & & & & & 0.13 & 0.46 & 532 & 0.11 & 0.42 & 446 & 0.439 & 0.05 \\
\hline $\begin{array}{l}\text { Times admitted to accident } \\
\text { and emergency department }\end{array}$ & & & & & & & & & 0.15 & 0.38 & 57 & 0.08 & 0.32 & 42 & 0.319 & 0.20 \\
\hline $\begin{array}{l}\text { Times required an } \\
\text { ambulance }\end{array}$ & & & & & & & & & 0.04 & 0.45 & 503 & 0.05 & 0.42 & 435 & 0.683 & -0.02 \\
\hline Times admitted to hospital & & & & & & & & & 0.05 & 0.23 & 525 & 0.05 & 0.21 & 444 & 0.828 & 0.00 \\
\hline $\begin{array}{l}\text { Elective admissions to } \\
\text { hospital }\end{array}$ & & & & & & & & & 0.95 & 0.69 & 21 & 0.84 & 0.68 & 18 & 0.637 & 0.16 \\
\hline $\begin{array}{l}\text { Non-elective admissions to } \\
\text { hospital }\end{array}$ & & & & & & & & & 0.36 & 1.88 & 210 & 0.37 & 0.62 & 17 & 0.964 & -0.01 \\
\hline $\begin{array}{l}\text { Other times visited hospital } \\
\text { (not including above) }\end{array}$ & & & & & & & & & 0.34 & 0.91 & 523 & 0.32 & 1.05 & 439 & 0.877 & 0.02 \\
\hline \multicolumn{17}{|l|}{ Social cognition variables } \\
\hline \multicolumn{17}{|l|}{ Fruit and vegetables } \\
\hline Affective attitude & 5.96 & 1.28 & 658 & 5.94 & 1.18 & 560 & 0.823 & -0.02 & 6.03 & 1.17 & 545 & 6.07 & 1.28 & 454 & 0.607 & 0.03 \\
\hline Cognitive attitude & 6.78 & 0.77 & 656 & 6.75 & 0.71 & 559 & 0.380 & -0.04 & 6.82 & 0.70 & 543 & 6.77 & 0.64 & 454 & 0.212 & -0.07 \\
\hline Subjective norm & 6.14 & 1.28 & 658 & 6.13 & 1.18 & 559 & 0.885 & -0.01 & 6.26 & 1.17 & 545 & 6.21 & 1.07 & 455 & 0.495 & -0.04 \\
\hline Descriptive norm & 2.67 & 1.28 & 659 & 2.63 & 1.18 & 561 & 0.557 & -0.03 & 2.72 & 1.17 & 545 & 2.69 & 1.28 & 455 & 0.644 & -0.02 \\
\hline Self-efficacy & 5.63 & 1.54 & 658 & 5.50 & 1.66 & 561 & 0.170 & -0.08 & 5.83 & 1.40 & 545 & 5.57 & 1.49 & 456 & 0.005 & -0.18 \\
\hline Perceived control & 5.43 & 1.54 & 659 & 5.42 & 1.66 & 560 & 0.953 & -0.01 & 5.63 & 1.63 & 544 & 5.56 & 1.49 & 456 & 0.479 & -0.04 \\
\hline Intention & 4.68 & 1.79 & 657 & 4.79 & 1.65 & 558 & 0.235 & 0.06 & 4.93 & 1.63 & 540 & 4.96 & 1.71 & 455 & 0.843 & 0.02 \\
\hline Planning & 5.00 & 1.79 & 657 & 4.93 & 1.66 & 560 & 0.564 & -0.04 & 5.14 & 1.63 & 544 & 5.14 & 1.71 & 455 & 0.998 & 0.00 \\
\hline \multicolumn{17}{|l|}{ Physical activity } \\
\hline Affective attitude & 5.65 & 1.54 & 659 & 5.61 & 1.66 & 560 & 0.680 & -0.03 & 5.73 & 1.40 & 545 & 5.76 & 1.49 & 454 & 0.742 & 0.02 \\
\hline Cognitive attitude & 6.80 & 0.77 & 658 & 6.77 & 0.71 & 561 & 0.539 & -0.04 & 6.84 & 0.70 & 544 & 6.77 & 0.64 & 454 & 0.105 & -0.10 \\
\hline Subjective norm & 6.07 & 1.28 & 659 & 6.09 & 1.18 & 560 & 0.821 & 0.02 & 6.20 & 1.17 & 545 & 6.21 & 1.07 & 455 & 0.894 & 0.01 \\
\hline Descriptive norm & 3.68 & 1.28 & 659 & 3.78 & 1.42 & 561 & 0.202 & 0.07 & 3.86 & 1.40 & 544 & 3.83 & 1.28 & 454 & 0.706 & -0.02 \\
\hline Self-efficacy & 5.68 & 1.54 & 658 & 5.57 & 1.42 & 561 & 0.198 & -0.07 & 5.88 & 1.40 & 545 & 5.71 & 1.49 & 456 & 0.049 & -0.12 \\
\hline Perceived control & 5.60 & 1.54 & 658 & 5.59 & 1.66 & 561 & 0.886 & -0.01 & 5.73 & 1.63 & 545 & 5.65 & 1.49 & 455 & 0.441 & -0.05 \\
\hline Intention & 5.34 & 1.80 & 660 & 5.43 & 1.66 & 560 & 0.364 & 0.05 & 5.32 & 1.87 & 545 & 5.47 & 1.71 & 455 & 0.158 & 0.08 \\
\hline Planning & 4.98 & 1.80 & 658 & 5.00 & 1.89 & 560 & 0.902 & 0.01 & 5.22 & 1.87 & 544 & 5.19 & 1.71 & 455 & 0.788 & -0.02 \\
\hline \multicolumn{17}{|l|}{ Binge drinking } \\
\hline Affective attitude & 3.17 & 1.80 & 659 & 3.08 & 1.89 & 560 & 0.379 & 0.05 & 3.02 & 1.87 & 545 & 3.03 & 1.71 & 455 & 0.944 & -0.01 \\
\hline Cognitive attitude & 1.82 & 1.03 & 658 & 1.68 & 0.95 & 561 & 0.019 & 0.14 & 1.68 & 0.93 & 543 & 1.68 & 1.07 & 454 & 0.998 & 0.00 \\
\hline Subjective norm & 2.43 & 1.54 & 659 & 2.33 & 1.42 & 560 & 0.275 & 0.07 & 2.36 & 1.40 & 543 & 2.40 & 1.49 & 455 & 0.694 & -0.03 \\
\hline Descriptive norm & 5.63 & 1.28 & 659 & 5.60 & 1.42 & 561 & 0.616 & 0.02 & 5.57 & 1.17 & 545 & 5.71 & 1.28 & 455 & 0.071 & -0.11 \\
\hline Self-efficacy & 6.13 & 1.28 & 658 & 6.16 & 1.42 & 561 & 0.783 & -0.02 & 6.20 & 1.40 & 545 & 6.15 & 1.28 & 456 & 0.574 & 0.04 \\
\hline Perceived control & 5.68 & 1.80 & 659 & 5.76 & 1.66 & 561 & 0.400 & -0.05 & 5.82 & 1.63 & 543 & 5.89 & 1.71 & 455 & 0.461 & -0.04 \\
\hline Intention & 3.38 & 1.80 & 658 & 3.24 & 1.89 & 558 & 0.210 & 0.08 & 3.28 & 1.86 & 543 & 3.19 & 1.92 & 455 & 0.434 & 0.05 \\
\hline
\end{tabular}


Table 4 Estimated marginal means, percentages, sample sizes, standard deviations and p values for secondary outcomes (Continued)

\begin{tabular}{lccccccccccccccccc}
\hline Planning & 4.87 & 2.05 & 657 & 4.79 & 2.13 & 560 & 0.478 & -0.04 & 4.99 & 2.10 & 543 & 4.84 & 2.13 & 455 & 0.244 & -0.07 \\
Smoking & & & & & & & & & & & & & & & & & \\
Affective attitude & 1.55 & 1.28 & 658 & 1.54 & 1.18 & 560 & 0.940 & 0.01 & 1.32 & 0.70 & 537 & 1.35 & 0.69 & 525 & 0.491 & -0.04 \\
Cognitive attitude & 1.27 & 0.77 & 658 & 1.26 & 0.71 & 559 & 0.648 & 0.01 & 1.59 & 1.17 & 545 & 1.53 & 1.28 & 455 & 0.398 & 0.05 \\
Subjective norm & 1.31 & 0.77 & 658 & 1.30 & 0.71 & 559 & 0.854 & 0.01 & 1.35 & 0.93 & 545 & 1.35 & 0.85 & 455 & 0.940 & 0.00 \\
Descriptive norm & 4.01 & 1.54 & 658 & 3.98 & 1.42 & 561 & 0.709 & 0.02 & 4.04 & 1.40 & 545 & 4.16 & 1.49 & 455 & 0.169 & -0.08 \\
Self-efficacy & 6.53 & 2.05 & 658 & 6.52 & 1.18 & 561 & 0.899 & 0.01 & 6.48 & 1.17 & 545 & 6.55 & 1.28 & 456 & 0.384 & -0.06 \\
Perceived control & 5.46 & 2.05 & 659 & 5.33 & 1.89 & 561 & 0.272 & 0.07 & 5.52 & 1.86 & 543 & 5.54 & 1.92 & 454 & 0.864 & -0.01 \\
Intention & 1.56 & 1.28 & 658 & 1.58 & 1.42 & 558 & 0.826 & -0.01 & 1.67 & 1.40 & 545 & 1.59 & 1.28 & 455 & 0.322 & 0.06 \\
Planning & 5.86 & 2.05 & 658 & 5.79 & 1.89 & 560 & 0.524 & -0.04 & 5.81 & 1.86 & 542 & 5.69 & 1.92 & 454 & 0.342 & -0.06 \\
\hline
\end{tabular}

effect on the biochemical marker of alcohol use (fatty acid ethyl esters) at 6-month follow-up, with lower levels of alcohol use observed among participants in the intervention versus control condition. The intervention also had significant effects on three social cognitive variables. Participants in the intervention condition had a more negative cognitive attitude towards binge drinking than participants in the control condition at 1-month followup. However, contrary to expectations, participants in the intervention condition had lower self-efficacy scores for fruit and vegetable intake and physical activity than participants in the control condition at 6-month followup. The effect of the intervention on all other secondary outcomes (e.g., recreational drug use, health status, BMI, health service usage) was non-significant.

\section{Discussion}

This paper reports the results of a repeat randomized controlled trial of a theory-based online health behaviour intervention delivered during the transition from school to university. The original trial [16] was compromised by a number of study limitations that resulted in low levels of engagement with the intervention. As a result, a number of changes were instigated in the repeat trial to increase engagement, including a shorter baseline questionnaire (to reduce participant fatigue), the use of the LifeGuide open-source software platform [18] to deliver the intervention (to improve the experience of engaging with the intervention), and a streamlined modular structure (so that participants could access the intervention material more quickly). These changes were successful in increasing engagement with the intervention. Thus, $85 \%$ of participants in the intervention condition completed the self-affirmation manipulation compared with $52 \%$ in the original trial, $72 \%$ accessed at least one health message (and $47 \%$ accessed health messages for all four health behaviours) compared with the $35 \%$ who accessed the health messages in the original trial, and $41 \%$ formed at least one implementation intention (and $29 \%$ formed implementation intentions for all four health behaviours) compared with $1 \%$ in the original trial.

Despite increased engagement, the primary analyses indicated that the effect of the intervention on the targeted health behaviours at 6-month follow-up was non-significant, although the effect on fruit and vegetable intake approached significance. Moreover, a perprotocol analysis revealed that participants who engaged with the intervention reported consuming significantly more portions of fruit and vegetables at 6-month followup than participants in the control condition. The original intervention was found to have a significant effect on smoking status at 6-month follow-up, which was primarily due to preventing non-smokers from starting smoking at university. This effect was not replicated in the repeat trial, although the direction of the effect was consistent. In addition, secondary analyses indicated that significantly fewer participants in the intervention condition reported that they had smoked at university (including a puff or socially) than those in the control condition. The intervention was also found to have a significant impact on the biochemical marker of alcohol use at 6-month follow-up, with lower levels of alcohol observed among participants in the intervention than in the control condition. This effect was in the same direction as the non-significant effect of the intervention on alcohol consumption in the primary analyses. Notably, participants who provided a hair sample did not differ from the rest of the baseline sample in their health behaviour.

The effect sizes for the intervention on the primary outcomes in the repeat trial were comparable to those found in the original trial, although two differences approached significance. The effect size for intervention on fruit and vegetable intake was marginally larger in the repeat trial, whereas the effect size for smoking status was marginally larger in the original trial. Overall, the effect sizes found in both trials were very small [33] and, with the exception of smoking status in the original trial, smaller than 
those typically found in online health behaviour change interventions [8].

There are several potential reasons for the relatively weak effects obtained in the repeat trial (which also relate to the original trial). First, the intervention sought to target four health behaviours in a single intervention. Webb et al. [8] reported that online interventions that target several health behaviours typically have smaller effects $(d=0.12)$ than those that target a single health behaviour $(d=0.17)$. A focus on several health behaviours may dilute the effect of the intervention on individual behaviours if participants choose only to change a single health behaviour. For example, an intervention targeting multiple health behaviours might help some participants to increase their fruit and vegetable intake and others to increase their levels of physical activity. Second, although levels of engagement with the intervention were increased in the repeat trial, they were still relatively low. Again, this may have been due to the focus on several health behaviours. For example, while $72 \%$ of participants in the intervention condition accessed at least one health message, only $47 \%$ accessed health messages for all four health behaviours. Similarly, while $41 \%$ formed at least one implementation intention, only $29 \%$ formed implementation intentions for all four health behaviours. Furthermore, there was some evidence that engagement moderated intervention effectiveness, with the per-protocol analyses revealing a significant effect of the intervention on fruit and vegetable intake (although other effects remained non-significant). Third, the baseline sample recruited into the study reported engaging in the recommended health behaviours at a similar or greater extent than 16-24-year-olds in England [2, 3]. It is therefore possible that the lack of positive effects for the intervention could be due to a 'ceiling effect' at baseline. However, analysis of participants in the control condition revealed that consumption of fruit and vegetables decreased, and alcohol consumption and the number of current smokers increased, after starting university, which would negate this explanation for the null findings. ${ }^{1}$

A number of limitations should be noted. First, in line with the original trial and the study protocol $[9,16]$, the effect of the intervention on the primary outcomes was assessed using an intention-to-treat approach in which missing data at 6-months were imputed from the 1month follow-up data by carrying the last observation forward. The use of this procedure has been criticized as it may introduce bias in the results (in either direction) and lead to overly narrow confidence intervals [32]. In particular, it assumes that students' health behaviour would have remained stable from 1- to 6-month followup. Analysis of the control condition revealed changes in three of the four health behaviours between these two time points. However, as recommended by Altman [32], repeating the primary analyses without data imputation produced consistent results.

Second, the primary outcomes were assessed by selfreport. To try to address this issue, we also sought to identify biochemical markers of alcohol and smoking behaviour by analyzing samples of hair provided by participants. Only 213 (8\%) of participants recruited into the trial participated in this aspect of the study. While highly selective, these participants were not found to differ from the rest of the sample on baseline measures of health behaviour. It should also be noted that there were no biochemical markers of fruit and vegetable intake, which precludes verification of the significant effect of the intervention effect found in the perprotocol analyses. Moreover, the fact that per-protocol analyses only included participants in the intervention condition who completed all intervention tasks (i.e., those who completed the self-affirmation profile, viewed a health message and formed an implementation intention) is likely to have introduced bias. As a result, the significant effect of the intervention on fruit and vegetable intake found in the per-protocol analyses should be treated with caution.

Third, although the response to the initial invitation emails was higher $(48.1 \%$ vs. $31.3 \%)$ in the repeat trial (primarily due to the invitation emails being sent out a week earlier and the use of a shorter baseline questionnaire), recruitment into the study was still relatively low. This was despite the use of a number of techniques that have been shown to increase response rates to online surveys, including the use of incentives and reminders [34]. In addition, participation in the repeat trial was higher among women, non-UK students and younger students, which may limit the generalizability of the findings.

Fourth, attrition was higher in the repeat trial, with only $55.8 \%$ of participants providing follow-up data (vs. $76.6 \%$ in the original trial), and was higher in the intervention arm than the control arm, which may have been a consequence of the longer time that participants spent engaging with the intervention in the repeat trial. Participants who dropped out were found to have a lower $\mathrm{BMI}$ and stronger intentions to eat fruit and vegetables at baseline than participants who provided follow-up data. Participants who dropped out were also less likely to be British, white or female.

Fifth, the intervention used three theory-based techniques (i.e., self-affirmation, theory-based messages and implementation intentions), which were combined in a single intervention. Such a design precludes the identification of the active, or redundant, ingredients of an intervention. Future work could employ full factorial designs to assess the impact of different combinations of 
these techniques on health behaviour. There is some evidence that combining self-affirmation manipulations and implementation intentions has a beneficial effect on fruit and vegetable intake [35], although other research suggests that this combination may have a detrimental effect on physical activity [36].

\section{Conclusions}

A repeat trial of an online theory-based health behaviour intervention for new university students found no significant effects on the primary outcome measures at 6month follow-up, although three positive effects were observed in ancillary analyses. First, a per-protocol analysis showed a significant effect of the intervention on fruit and vegetable intake. Second, secondary analyses revealed that significantly fewer participants in the intervention condition had smoked while at university than in the control condition. Third, analysis of hair samples demonstrated significantly lower use of alcohol among participants in the intervention condition than in control condition. Nonetheless, the overall effect of the intervention on the targeted health behaviours was relatively weak. This may have been due, in part, to the focus of the intervention on multiple, rather than, single health behaviours. Future interventions targeting the health behaviour of new university students might focus on smoking behaviour, given the positive intervention effects in the original and repeat trials and the important impact that smoking has on health outcomes.

\section{Endnote}

${ }^{1}$ Analysis of participants in the control condition revealed significant effects of time on portions of fruit and vegetables consumed, $F(2,932)=30.17, P<0.001$, units of alcohol consumed, $F(2,910)=48.83, P<0.001$, and number of current smokers, Cochran's $Q(2)=$ 27.94, $P<0.001$. Post-hoc analyses indicated that the consumption of fruit and vegetables decreased from baseline $($ mean $=4.41, \mathrm{SD}=1.92)$ to 1 -month follow-up $($ mean $=3.70, \mathrm{SD}=1.90)$ and then increased from 1 - to 6-month follow-up $($ mean $=3.98, \quad \mathrm{SD}=2.07)$, alcohol consumption increased from baseline (mean $=6.72$, $\mathrm{SD}=9.79)$ to 1 -month follow-up (mean $=11.99, \mathrm{SD}=$ 14.72) and then decreased from 1- to 6-month follow-up (mean $=10.57, \mathrm{SD}=12.97)$, and the number of current smokers increased from baseline (3.08 \%) to 1 -month follow-up (5.45 \%) and also from 1- to 6month follow-up $(9.00 \%)$. Differences between all time points were significant for all three of the health behaviours. Physical activity levels remained stable over time, $F(2,920)=0.99, P=0.37$.

\section{Abbreviations}

ANCOVA: analysis of covariance; AUDIT: Alcohol Use Disorders Identification Test; BMl: body mass index.

\section{Competing interests}

The authors declare that they have no competing interests.

\section{Authors' contributions}

DC was responsible for the data analysis and wrote the first draft of the method and results sections. TE was responsible for the design of the intervention and the programming and administration of the baseline questionnaire and intervention on LifeGuide. PN was responsible for the administration of the follow-up questionnaires. He also wrote the first draft of the abstract, introduction and discussion and finalized the paper following feedback from other authors. PN, PS, PRH, TLW and TE were responsible for the development of the intervention material. SAJ was responsible for the statistical analysis plan. $A B$ and $C T$ advised on the choice of measures and were responsible for the health economic evaluation. AP was responsible for the indirect estimation of recreational drug use. DN and IS were responsible for the biochemical analysis of the hair samples. All authors commented on drafts and approved the final version of the paper.

\section{Acknowledgements}

The study was funded by the UK National Prevention Research Initiative Phase 4 (grant number: MR/J0004501/1) which includes the following Funding Partners (in alphabetical order): Alzheimer's Research Trust, Alzheimer's Society, Biotechnology and Biological Sciences Research Council, British Heart Foundation, Cancer Research UK, Chief Scientist Office, Scottish Government Health Directorate, Department of Health, Diabetes UK,

Economic and Social Research Council, Health and Social Care Research and Development Division of the Public Health Agency (HSC and R\&D Division), Medical Research Council, The Stroke Association, Wellcome Trust, Welsh Assembly Government and World Cancer Research Fund.

\section{Author details}

${ }^{1}$ Department of Psychology, University of Sheffield, Western Bank, Sheffield S10 2TP, UK. ${ }^{2}$ School of Psychological Science, University of Manchester, Oxford Road, Manchester M13 9PL, UK. ${ }^{3}$ Psychology Department, University of North Carolina, 323 Davie Hall, Chapel Hill, NC 27599-3270, USA. ${ }^{4}$ School of Psychology, University of Sussex, Falmer, Brighton BN1 9QH, UK. ${ }^{5}$ School of Health and Related Research, University of Sheffield, Regent Court, Sheffield S1 4DA, UK. '5 School of Life Sciences, Pharmacy and Chemistry, Kingston University, Penrhyn Road, Kingston upon Thames KT1 2EE, UK.

Received: 3 July 2015 Accepted: 27 November 2015

Published online: 07 December 2015

\section{References}

1. Parliamentary Office of Science and Technology. Health behavior. http:// www.parliament.uk/documents/post/postpn283.pdf. Accessed 2 June 2015

2. Heath and Social Care Information Centre. Health Survey for England 2012. http://www.hscic.gov.uk/catalogue/PUB13218. Accessed 2 June 2015.

3. Heath and Social Care Information Centre. Health survey for England 2013. http://www.hscic.gov.uk/searchcatalogue?productid=16571\& infotype $=0 \% 2 \mathrm{fS}$ urvey \&sort=Most+recent\&size $=10 \&$ page $=1$ \#top. Accessed 2 June 2015.

4. Schuit AJ, van Loon AJM, Tijhuis M, Ocké M. Clustering of lifestyle risk factors in a general adult population. Prev Med. 2002;35:219-24.

5. Poortinga $W$. The prevalence and clustering of four major lifestyle risk factors in an English adult population. Prev Med. 2007:44:124-8.

6. Jemmott JB, Jemmott LS, Leary AO, Ngwane Z, Icard L, Bellamy S, et al. Cognitive-behavioral health-promotion intervention increases fruit and vegetable consumption and physical activity among South African adolescents. Psychol Health. 2011;26:167-85.

7. Zhang Y, Cooke R. Using a combined motivational and volitional intervention to promote exercise and healthy dietary behaviour among undergraduates. Diab Res Clin Pract. 2012;95:215-23.

8. Webb TL, Joseph J, Yardley L, Michie S. Using the internet to promote health behavior change. J Med Internet Res. 2010;12, e4.

9. Epton T, Norman P, Sheeran P, Harris PR, Webb TL, Ciravegna F, et al. A theory-based online health behaviour intervention for new university students: study protocol. BMC Public Health. 2013;13:107.

10. Lawson PJ, Flocke SA. Teachable moments for health behavior change: a concept analysis. Patient Educ Couns. 2009;76:25-30. 
11. Heatherton TF, Nichols PA. Personal accounts of successful versus failed attempts at life change. Pers Soc Psychol Bull. 1994;20:664-75.

12. UCAS. End of cycle report 2014. https://www.ucas.com/sites/default/files/ 2014-undergraduate-end-of-cycle-report.pdf. Accessed 2 June 2015.

13. Epton $T$, Harris PR, Kane R, van Koningsbruggen GM, Sheeran P. The impact of self-affirmation on health behavior change: a meta-analysis. Health Psychol. 2015;34:187-96.

14. Epton T, Norman P, Harris P, Webb T, Snowsill A, Sheeran P. Development of theory-based health messages: three-phase programme of formative research. Health Promot Int. 2015;30:756-68.

15. Gollwitzer PM, Sheeran P. Implementation intentions and goal achievement: a meta-analysis of effects and processes. Adv Exp Soc Psychol. 2006;38:69-119.

16. Epton T, Norman P, Dadzie AS, Harris PR, Webb TL, Sheeran P, et al. A theory-based online health behaviour intervention for new university students (U@Uni): results from a randomised controlled trial. BMC Public Health. 2014;14:563.

17. Kruger J, Brennan A, Strong M, Norman P, Epton T, Thomas C. The costeffectiveness of a theory-based online health behaviour intervention for new university students: an economic evaluation. BMC Public Health. 2014;14:1011.

18. Williams S, Yardley L, Weal M, Wills G. Introduction to LifeGuide: opensource software for creating online interventions for health care, health promotion and training. Luxembourg: Proceedings Med-e-tel, Global Telemedicine and eHealth Updates, Knowledge Resources; 2010. p. 187-90

19. Sherman DK, Bunyan DP, Creswell JD, Jaremka LM. Psychological vulnerability and stress: the effects of self-affirmation on sympathetic nervous system responses to naturalistic stressors. Health Psychol. 2009;28:55-62.

20. Ajzen I. Attitudes, personality and behavior. Milton Keynes: Open University Press; 1988.

21. Oettingen $G$, Hönig G, Gollwitzer PM. Effective self-regulation of goa attainment. Int J Educ Res. 2002;33:705-32.

22. Cappuccio FP, Rink E, Perkins-Porras L, Hilton S, Steptoe A. Estimation of fruit and vegetable intake using a two-item dietary questionnaire: a potential tool for primary health workers. Nutr Metab Cardiovasc Dis. 2003;13:12-9.

23. Booth ML. Assessment of physical activity: an international perspective. Res Q Exercise Sport. 2000;71:s114-20.

24. Gmel G, Rehm J. Measuring alcohol consumption. Contemp Drug Probl. 2004:31:467-540

25. Babor TF, Higgins-Biddle JC, Saunders JB, Nonteiro MG. The Alcohol Use Disorders Identification Test. Geneva: World Health Organization; 2001.

26. Rabin $\mathrm{R}$, de Charro F. EQ-5D. A measure of health status from the EuroQol Group. Ann Med. 2001;23:337-43.

27. Dolan P, Gudex C, Kind P, Williams A. A social tariff for EuroQoL: results from a UK general population survey. Discussion Paper No. 138. In Centre for Health Economics. York: University of York; 1995.

28. Petróczi A, Cross P, Taft H, Shah S, Deshmukh N, Nepusz T, et al. New nonrandomised model to assess the prevalence of discriminating behaviour: a pilot study on Mephedrone. Subst Abuse Treat Prev Policy. 2011;6:20.

29. Nepusz T, Petróczi A, Naughton D, Epton T, Norman P. Estimating the prevalence of socially sensitive behaviours: attributing guilty and innocent noncompliance with the single sample count method. Psychol Methods. 2014;19:334-55.

30. Julious SJ, Mullen MA. Issues with using baseline in last observation carried forward analysis. Pharm Stat. 2008;7:146.

31. International Conference on Harmonisation E9 Expert Working Group. ICH Harmonised tripartite guideline. Statistical principles for clinical trials. Stat Med. 1999;18:1905-42.

32. Altman DG. Missing outcomes in randomized trials: addressing the dilemma. Open Med. 2009;3:51-3.

33. Cohen J. A power primer. Psychol Bull. 1992;112:155-9.

34. Edwards PJ, Roberts I, Clarke MJ, DiGuiseppi C, Wentz R, Kwan I, et al. Methods to increase response to postal and electronic questionnaires. Cochrane Database of Syst Rev. 2009, 3: MR000008. http://www.cochrane. org/MR000008/METHOD_methods-to-increase-response-to-postal-andelectronic-questionnaires

35. Harris PR, Brearley I, Sheeran P, Barker M, Klein WMP, Creswell JD, et al. Combining self-affirmation with implementation intentions to promote fruit and vegetable consumption. Health Psychol. 2014:33:729-36.

36. Jessop DC, Sparks P, Buckland N, Harris PR, Churchill S. Combining selfaffirmation and implementation intentions: evidence of detrimental effects on behavioral outcomes. Ann Behav Med. 2014:47:137-47.

\section{Submit your next manuscript to BioMed Central and we will help you at every step:}

- We accept pre-submission inquiries

- Our selector tool helps you to find the most relevant journal

- We provide round the clock customer support

- Convenient online submission

- Thorough peer review

- Inclusion in PubMed and all major indexing services

- Maximum visibility for your research

Submit your manuscript at www biomedcentral com/submit 